CORRIGENDUM

\title{
Dispersal in microbes: fungi in indoor air are dominated by outdoor air and show dispersal limitation at short distances
}

\author{
Rachel I Adams, Marzia Miletto, John W Taylor and Thomas D Bruns
}

The ISME Journal (2013) 7, 1460; doi:10.1038/ismej.2013.84

Correction to: The ISME Journal (2013) 7, 1262-1273; doi:10.1038/ismej.2013.28; published online 21 February 2013

Since the publication of this article, the authors have noticed an error on Page 4, namely that the accession number contained within the first paragraph had been incorrectly listed as SRA059067 instead of SRA059097. The correct sentence is shown here.

Sequences were run on $1 / 8$ th of a 454 FLX Titanium pico-titer plate at the Duke Institute for
Genomic Sciences and Policy (Durham, NC, USA) and submitted to the National Center for Biotechnology Information Sequence Read Archive under accession number SRA059097.

The error has now been rectified, and the correct article appears in this issue. The html and online pdf versions have also been rectified, and now carry the correct paper.

The authors would like to apologize for any inconvenience this may have caused. 